\title{
Guest editorial for special issue on IMS modeling and simulation
}

\author{
Samar K. Guharay ${ }^{1}$
}

Published online: 19 August 2015

(C) Springer-Verlag Berlin Heidelberg 2015

As an effective and efficient analytical device ion mobility spectrometry (IMS) is routinely used in many critical trace detection applications. The role of IMS is steadily realized in many emerging applications as well, especially in biological/medical sciences, for example, in exploration of complex metabolomics, structural proteomics, etc. Increasing accuracy of mobility values, manipulating drift medium and reaction kinetics, enhancing sensitivity both algorithmically and by improved electronics, and above all, miniaturization with high performance, form some of the key topics for advancing the science and technology of IMS. New problems continue rejuvenating researchers and engineers to identify key questions, derive answers through systematic scientific studies and advance the boundary of technological development. While different configurations of this mobility-based device have been realized as a stand-alone technology, these devices are interfaced or hyphenated with other orthogonal sensing modalities for much enhanced performance. In all of these explorations modeling and simulation (M\&S) and theoretical work act as an underpinning tool to develop new design concepts, build their operational paradigms, and optimize performance metrics.

It is well recognized that IMS operation includes several distinct steps. Broadly speaking, ionization, pulsing/ gating, ion transport, ion detection, and algorithm for

Samar K. Guharay

sguharay@mitre.org

1 The MITRE Corporation, 7515 Colshire Dr., McLean, VA 22102, USA data exploitation constitute the key elements of IMS operation. While significant progress has been made in answering many questions, the complexity of each of these problems is exacerbated due to an overarching need of miniaturization without compromising performance metrics. Some of the key questions pertain to the mechanism of the reactant and the product ion formation for different ionization processes, effective gating and injection of charges from the ionization to drift region, ion-ion and ion-neutral interactions, ion diffusion, coulomb interactions and charge control, induced charge, screening of Faraday plate, and amplifier characteristics. Critical problems in these areas are being addressed by mobilizing expertise from all domains of science and engineering as well as in mathematics and statistics. In this vein, modeling and simulation, if sufficiently validated, can be very effectively utilized to gain insight into the problems and obtain guidance to design experiments. In further advancing the utility of the technology it will be important to undertake a modeling and simulation task integrating the end-toend process steps which will help building the overall system link budget. Accurately capturing the phenomenology governing each step that entails acquiring samples from a target, ionizing and transporting through IMS and finally detecting analyte ions will form the crux of the effort.

While this special issue by no means encompasses the entire span of modeling and simulation (M\&S) activities in IMS, each article presents a scholarly approach to problems of significant current interest and demonstrates the merit of M\&S. This special issue is offered to the community with the hope that it will provide a compendium sampling some of the areas of current interests on IMS modeling and simulation. 
Acknowledgments The Guest editor would like to thank the authors for their very valuable contribution and the reviewers for their support and time. The sustained encouragement and support of Dr. Steffen Pauly, Executive Editor Chemistry, Springer and of the Editors-in-Chief of the International Journal for Ion Mobility Spectrometry is thankfully acknowledged. Special thanks are due to Prof. Dr. Jörg Ingo Baumbach for meticulously implementing the review process and helping meet the targeted milestone to publish this special issue. The affiliation with The MITRE Corporation is provided for identification purposes only, and is not intended to convey or imply MITRE's concurrence with, or support for, the positions, opinions or viewpoints expressed in this Guest editorial introduction. 